\title{
Routine antibiotic therapy in the management of the local inflammatory swelling in venomous snakebites: results of a placebo-controlled study
}

\author{
SAM Kularatne ${ }^{1}$, PVR Kumarasiri ${ }^{2}$, SKC Pushpakumara' ${ }^{1}$, WP Dissanayaka ${ }^{3}$, H Ariyasena ${ }^{3}$, IB Gawarammana ${ }^{1}$ \\ and $\mathbf{N}$ Senanayake ${ }^{1}$
}

\begin{abstract}
Objective To evaluate the value of routine antibiotic therapy in the management of the local swelling of patients with venomous snakebites.

Methods A prospective, placebo-controlled study at the General Hospital, Anuradhapura, Sri Lanka where 144 patients with envenoming and local swelling in the leg were allocated to receive either antibiotics (Group $A=$ test group) or placebo (Group B = controls). Benzyl penicillin 2 mega units intravenously 6 hourly and metronidazole $500 \mathrm{mg}$ by intravenous infusion 8 hourly for 5 days from the first day of the bite were given to Group A. Ethical committee approval was obtained from the Committee of General Hospital, Anuradhapura.
\end{abstract}

Main outcome measures Circumference difference between the affected limb and the normal limb, length of the swelling measured in centimetres, and the physical characteristics of the local swelling of both groups were compared.

Results Group A had 69 patients and the Group B 75. The mean circumference difference (MCD) of the leg between the groups showed no significant difference for 4 days $(P>0.05)$, except at the site of the bite on the third day when the Group B showed a significant improvement $(p=0.02)$. There was no significant difference in the length of the local swelling or the score of physical characteristics between the two groups $(P>$ 0.05 ). The proportions of recovery of the local swelling on the fourth and fifth day had no significant difference between the groups $(P>0.05)$.

Conclusion The routine use of antibiotics (penicillin and metronidazole) does not seem to be of value in reducing the local inflammatory swelling in venomous snakebite.

\section{Introduction}

Venomous snakebite is an important problem in Sri Lanka [1]. Most bites are caused by Russell's viper (Daboia russelli russelli), hump-nosed viper (Hypnale hypnale), cobra (Naja naja) and the common krait (Bungarus caeruleus ) [1-4]. The first three snakes are well known to produce local effects at the site of the bite,

${ }^{1}$ Department of Medicine, ${ }^{2}$ Department of Community Medicine, University of Peradeniya, Sri Lanka; ${ }^{3}$ General Hospital, Anuradhapura, Sri Lanka.

Correspondence: SAMK, e-mail: <samkul@sltnet.lk>. (Competing interests: none declared). Received 3 January 2005 and revised version accepted 13 May 2005. 
varying from mild swelling to severe necrosis[1-4]. There are two explanations for the mechanism of local swelling. The first is cytotoxin-mediated inflammation producing necrosis of tissues, and the second is infection caused by bacterial contamination of the bite from oral flora of the snake [5,6]. A bacteriological study of the venom and mouth cavities of wild Malayan pit vipers has isolated Gram-negative rods such as Enterobacter and Pseudomonas, and staphylococci and clostridia [7]. Hence, this study has recommended to prescribe gentamicin with benzylpenicillin in Malayan pit viper bite [7]. Clostridia are the predominant anaerobic bacteria cultured in some previous studies [8,9]. However, the value of antibiotics for local swelling has been disputed by other studies [10-12].

In Sri Lanka, the use of antibiotics for the local effects is popular in the management of snakebite. Penicillin and metronidazole is the widely used combination of antibiotics. The efficacy of this practice has not been tested scientifically. There is no doubt that the local swelling developing within a few hours after snakebite is due to cytotoxins in the venom, but it may be argued that the persistence and spread of the local swelling is due to secondary infection. There is no proof that the oral flora of snakes is capable of infecting the skin and subcutaneous tissues of previously healthy people, unlike in the case of the Komodo dragon (Varanus komodoebsis) whose highly toxic oral bacterial flora cause a lethal infection of the bite wound [6]. Hence the value of routine antibiotics in the management of the local inflammatory swelling is open to question.

In this placebo-controlled study, we evaluated the efficacy of the two most commonly used antibiotics in Sri Lanka in the management of the local inflammatory swelling in patients with venomous snakebite.

\section{Methods}

We recruited patients from the medical wards of the General Hospital, Anuradhapura, Sri Lanka from June to November 2003. Patients aged 12-70 years who were admitted within 12 hours after snakebite on the leg at or above the ankle joint with local swelling were eligible for inclusion. We excluded patients who were allergic to penicillin and those who had associated debilitating diseases such as diabetes mellitus, malignant disease and hepatic, renal and heart disease. Patients with severe local tissue necrosis or gangrene at the time of admission, those who had herbal or antiseptic applications locally, and those with local incisions were also excluded.

We obtained written informed consent from all the patients included in the study. For those who were not able to give consent, we sought permission from a parent, spouse or guardian. The patients were told that they were free to withdraw from the trial at any time if they wished and in case the swelling became worse they would be treated actively.

\section{Treatment}

On admission, all patients were assessed and given standard treatment for snakebite, such as resuscitation, intravenous line, and monitoring of vital parameters. Lyophilised polyvalent enzyme-refined Indian antivenom serum (AVS) was given when indicated. Those who developed allergic reactions to antivenom were treated with adrenaline. In addition, they received one of the following treatment regimens for the local swelling based on allocation to Group A or B; Group A: Benzylpenicillin + metronidazole; Benzylpenicillin 2 mega units intravenous 6 hourly and metronidazole $500 \mathrm{mg}$, intravenous infusion 8 hourly for duration of 5 days. Group B: Placebo isotonic saline $5 \mathrm{~mL}$, intravenous, 6 hourly and isotonic saline $100 \mathrm{~mL}$ intravenous infusion 8 hourly for 5 days.

One of the above treatment regimens was administered. The criteria for interventions outside the trial protocol were: (a) patients who requested to be discharged from the hospital because of improvement of the local swelling and recovery, (b) worsening of the local inflammatory swelling, and (c) when surgical intervention was needed due to development of necrosis and gangrene of the local inflammatory swelling.

\section{Allocation}

A standard formula was used to calculate the sample size [13]. The power calculation was based on the assumption that the antibiotics would reduce the local infection rate by $25 \%$ as there were no previous studies, and keeping the $\alpha$ and $\beta$ errors at $5 \%$ and $10 \%$. Accordingly, we planned to recruit 180 patients. Patients who fulfilled the eligibility criteria were allocated to receive one of the two treatment regimens. Treatment and placebo were placed in envelopes which were kept in a safe container at the investigating centre, the General Hospital, Anuradhapura.

Two teams, namely the treatment team and the investigating team were identified in the management of patients. The investigating team and the patients were blind to the treatment regimen. A member of the treatment team administered the treatment or the placebo. The investigating team monitored and assessed the stipulated parameters of local swelling. One trained member from the investigating team was assigned to evaluate the local swelling of all the patients in order to eliminate interobserver variability.

\section{Data collected}

\section{Patients}

The patient's age, gender, time of the bite, time from the bite to admission, dose of antivenom given, reactions to antivenom, previous treatment and other medical conditions were recorded. A history of allergy to drugs, especially penicillin was noted. The biting snake was identified. 


\section{Envenoming}

Severity of envenoming was assessed by a scoring system devised by the investigating team which was tested in a previous study (Table 1) [14]. The total severity score was calculated for each patient (range 1-10), and for each group (A,B) for comparison of the groups. A score from 1 to 3 was given according to involvement of each system, and these were summed to give a mean rank score.

\section{Table 1. Scoring system for assessing severity of envenoming}

A score from 1 to 3 was given according to involvement of each system, and these were summed to give a mean rank score.

Haemostasis

Positive whole blood clotting test at $20 \mathrm{~min}$ (WBCT20) 1

Positive repeated WBCT20

Spontaneous bleeding

Neurotoxicity

Weakness of eye and facial muscles

Generalised muscle weakness

Respiratory muscle weakness leading to mechanical ventilation

Renal

Elevated serum creatinine

Associated oliguria

Peritoneal dialysis in acute renal failure

Rhabdomyolysis

Tender muscles

Myoglobinuria

Above with hyperkalaemia

Cardiac

Presence of ECG changes

Hypotension, arrhythmia, ischaemic chest pain

\section{Assessment of local swelling}

1. Measured variables: Site of bite (definitive fang mark) in the affected limb and the corresponding site in the normal limb were marked with ink. The circumference of both limbs was measured in centimetres at the level of the site of bite and at $5 \mathrm{~cm}$ intervals up to $25 \mathrm{~cm}$ proximally. The difference in the circumference between the affected and normal limbs was calculated at each level. Proximal length of the local swelling was measured in centimetres from the site of bite in the affected limb.

2. Physical characteristics: The colour of the skin over the local swelling was given a score as follows: normal 1 , redness 2 , necrosis 3 , and gangrene 4 . Other associated features such as pain, tenderness, temperature, loss of sensation, absent distal pulse, distal ischaemia and enlarged regional lymph nodes were given one point each. The total score of physical characteristics was calculated for each patient.

These assessments were done on the first day after recruitment and daily thereafter for the next 5 days.

\section{Statistical analysis}

The data were computerised and analysed using the statistical software, SPSS version 10. The frequency distributions of each variable were checked to ensure correct data entry. Then the baseline variables (age, sex, the snake responsible, severity of envenoming, time taken to start the treatment, number of AVS vials used, and reactions to AVS) were compared between Group A and Group B to ensure comparable allocation. The differences in leg circumference and the mean differences in the length between the two groups were compared using the unpaired t-test, whereas the clinical signs were compared using either Chi-square or Fisher's exact test. The MannWhitney U-test was applied to analyse the physical characteristic scores of local swellings between the groups.

\section{Results}

We recruited 250 patients, but 106 were excluded as they had one or more exclusion criteria (Figure 1) Of the 144 patients who fulfilled the eligibility criteria, 69 received antibiotics (penicillin and metronidazole) and 75 received placebo. Patient characteristics such as age and gender, biting snake, dose of antivenom, reaction to antivenom, time difference from bite to hospitalisation and severity of envenoming are shown in Table 2. There was no significant difference between the two groups for any of these variables.

The mean difference in the circumference of affected leg and normal leg was compared between the Groups A and B for three sites ( Table 3). There was no significant difference between the groups on the 4 days of follow up except on the third day which showed improvement of

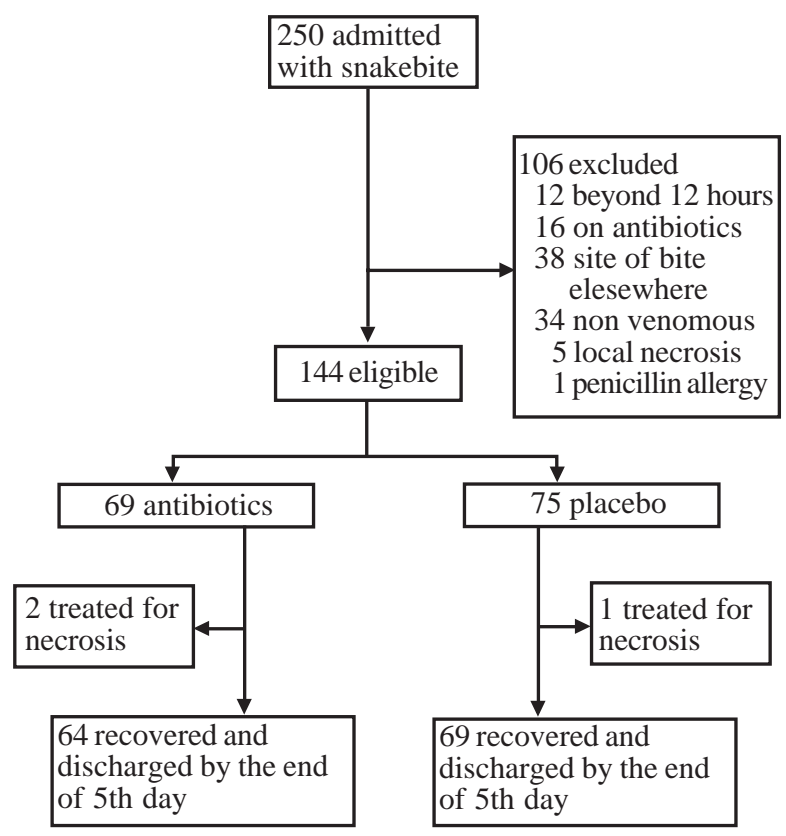

Figure 1. Trial flowchart. 
Table 2. Distribution of baseline data between the two groups

\begin{tabular}{|c|c|c|c|c|}
\hline \multicolumn{2}{|l|}{ Variable } & \multirow{2}{*}{$\begin{array}{c}\text { Test }(A) \\
54\end{array}$} & \multirow{2}{*}{$\frac{\text { Control }(B)}{57}$} & \multirow{2}{*}{$\begin{array}{l}\text { P value } \\
0.75^{*}\end{array}$} \\
\hline ex & Male & & & \\
\hline & Female & 15 & 18 & \\
\hline & Total & 69 & 75 & \\
\hline \multirow[t]{3}{*}{ Snake } & Viper & 54 & 51 & $0.166^{*}$ \\
\hline & Others & 15 & 24 & \\
\hline & Tota & 69 & 75 & \\
\hline Reactions to & No & 36 & 42 & $0.645^{*}$ \\
\hline \multirow[t]{2}{*}{ AVS } & Yes & 33 & 33 & \\
\hline & Total & 69 & 75 & \\
\hline \multicolumn{2}{|c|}{ Mean age (SD) } & $32.1(12.1)$ & $34.6(11.9)$ & $0.206 * *$ \\
\hline \multicolumn{2}{|c|}{ Mean number of } & & & \\
\hline \multicolumn{2}{|c|}{ AVS vials given (SD) } & $12.7(10.5)$ & $12.1(11.4)$ & $0.738 * *$ \\
\hline \multicolumn{2}{|c|}{ Mean time difference } & $4.5(3.9)$ & $4.6(2.7)$ & $0.90 * *$ \\
\hline \multicolumn{2}{|c|}{$\begin{array}{l}\text { Mean rank score of } \\
\text { severity of envenomation }\end{array}$} & 76.3 & 68.9 & $0.262 * * *$ \\
\hline
\end{tabular}

* Chi-square test, ** unpaired t-test, ***Mann-Whitny U-test

local swelling at the site in Group B. Similarly, the mean length of the local swelling was compared which showed no significant difference between the two groups during the 4 days ( Table 4). The physical characteristics of the local swelling between the two groups showed no significant difference during the 3 days of follow up (Table 5). At the end of the fourth day, $65.3 \%$ and $74.7 \%(\mathrm{p}=$ 0.22) of the patients in Group A and Group B had improvement of the local inflammatory swelling, and left the hospital. The corresponding values at the end of the fifth day were $91.3 \%$ and $93.3 \%(p=0.65)$ respectively. This difference was not statistically insignificant. Furthermore, three subjects (two in the test group and one in the control group) had worsening of the local swelling and early necrosis by the third day. They were treated surgically and with different antibiotics.

\section{Discussion}

We tested the value of antibiotics for the local swelling of snakebite as this is a routine practice in Sri Lanka.
Table 4. Mean length of the local swelling between two groups by days

\begin{tabular}{llllll}
\hline Day & \multicolumn{2}{l}{ Mean length $(S D)$ in cm } & 95\% CI** & P value* \\
& Test $(A)$ & Control $(B)$ & & & \\
\hline One & $14.67(10.17)$ & $15.27(9.93)$ & -3.913 and 2.713 & 0.72 \\
Two & $19.19(12.08)$ & $20.15(9.16)$ & -4.541 and 2.624 & 0.59 \\
Three & $19.05(12.93)$ & $21.12(11.97)$ & -6.223 and 2.069 & 0.32 \\
Four & $23.25(14.88)$ & $25.42(12.12)$ & -10.69 and 6.349 & 0.16 \\
\hline
\end{tabular}

$* \mathrm{p}$ value for $\mathrm{t}$-test, $* * 95 \%$ confidence interval

Table 5. Distribution of physical characteristics of local swelling between the two groups

\begin{tabular}{|c|c|c|c|c|c|}
\hline Day & Variable & $\operatorname{Test}(A)$ & Control (B) & Total & Pvalue \\
\hline \multirow[t]{6}{*}{ One } & Colour (+) & 67 & 74 & 141 & $0.61 * *$ \\
\hline & Colour $(\rightarrow$ & 2 & 01 & 03 & \\
\hline & Tenderness (+) & 57 & 68 & 125 & $0.15^{*}$ \\
\hline & Tenderness $(\rightarrow$ & 12 & 7 & 19 & \\
\hline & $\begin{array}{l}\text { Mean rank score } \\
\text { of physical } \\
\text { characteristics of }\end{array}$ & & & & \\
\hline & of local swelling & 70.5 & 74.4 & & $0.525 * * *$ \\
\hline \multirow[t]{6}{*}{ Two } & Colour (+) & 66 & 73 & 139 & $0.671 *$ \\
\hline & Colour $(\rightarrow$ & 03 & 02 & 05 & \\
\hline & Tenderness (+) & 39 & 44 & 83 & $0.79 *$ \\
\hline & Total & 69 & 75 & 144 & \\
\hline & $\begin{array}{l}\text { Mean rank score } \\
\text { of Physical }\end{array}$ & & & & \\
\hline & $\begin{array}{l}\text { Characteristics of } \\
\text { of local swelling }\end{array}$ & 73.4 & 71.6 & & $0.78^{* * *}$ \\
\hline \multirow[t]{6}{*}{ Three } & Colour $(+)$ & 62 & 71 & 133 & $0.278^{*}$ \\
\hline & Colour (-) & 7 & 4 & 11 & \\
\hline & Tenderness (+) & 8 & 13 & 21 & $0.35^{*}$ \\
\hline & Tenderness (-) & 59 & 61 & 120 & \\
\hline & $\begin{array}{l}\text { Mean rank score } \\
\text { of physical }\end{array}$ & & & & \\
\hline & $\begin{array}{l}\text { characteristics of } \\
\text { of local swelling }\end{array}$ & 62.9 & 60.6 & 0.699 & $* * *$ \\
\hline
\end{tabular}

*P value of Chi-square test, **P values of Fisher's exact test $* * *, \mathrm{P}$ value of Mann-Whitney U-test

Table 3. Mean circumference difference of legs between two groups by the days

\begin{tabular}{|c|c|c|c|c|c|c|}
\hline Day & Distance to the & Mean circur & erence $(S D)$ in $\mathrm{cm}$ & $95 \% C$ & $C I^{* *}$ & $P$ value* \\
\hline & bite site & TestA & Control(B) & & & \\
\hline One & At the site & $1.69(1.22)$ & $1.42(1.04)$ & 0.104 and & 0.642 & 0.15 \\
\hline & $10 \mathrm{~cm}$ & $0.86(0.83)$ & $0.80(0.78)$ & 0.203 and & 0.328 & 0.64 \\
\hline & $20 \mathrm{~cm}$ & $0.55(0.99)$ & $0.63(1.10)$ & -0.414 and & 0.277 & 0.69 \\
\hline Two & At the site & 2.09 (1.39) & $2.03(1.09)$ & -.350 and & 0.470 & 0.77 \\
\hline & $10 \mathrm{~cm}$ & $1.42(1.18)$ & $1.43(1.16)$ & -0.402 and & 0.374 & 0.94 \\
\hline & $20 \mathrm{~cm}$ & $0.98(1.13)$ & $1.14(1.36)$ & 0.573 and & 0.249 & 0.43 \\
\hline Three & At the site & $2.31(1.87)$ & $1.68(1.05)$ & ๑. 132 and & d 1.128 & 0.02 \\
\hline & $10 \mathrm{~cm}$ & $1.50(1.23)$ & $1.56(1.32)$ & 0.486 and & 0.365 & 0.78 \\
\hline & $20 \mathrm{~cm}$ & $1.05(1.32)$ & $1.12(1.43)$ & -0.528 and & 0.389 & 0.77 \\
\hline Four & At the site & $2.32(2.18)$ & $1.71(1.02)$ & -.484 and & 1.703 & 0.27 \\
\hline & $10 \mathrm{~cm}$ & $1.71(1.22)$ & 1.71 (1.17) & -0.747 and & 0.742 & 0.99 \\
\hline & $20 \mathrm{~cm}$ & $1.52(1.49)$ & $1.44(1.26)$ & -.788 and & 0.935 & 0.86 \\
\hline
\end{tabular}

*unpaired t-test, ** 95\% confidence interval 
One of the shortcomings of this study was that we were able only to recruit 144 patients due to practical difficulties. But this accounted for $80 \%$ of the intended 180 patients and we feel that the reduced number could not affect our results. We used a combination of two antibiotics to cover a broad range of pathogens, both aerobic and anaerobic, that are known to exist in the oral cavity of snake [7-9]. We refrained from using gentamicin as it has adverse effects on the kidney. We found that penicillin and metronidazole had no significant benefit $(\mathrm{p}>0.05)$ in reducing the severity of the local swelling compared with placebo. The local swelling followed its natural course of recovery irrespective of antibiotic therapy. Hence the use of a different combination of antibiotics or adding more antibiotics to the regimen may not be beneficial.

There are a number of studies done elsewhere with a similar research question. A prospective observational study has found a low incidence of wound infection following crotalid ( rattlesnake) bite and has recommended avoidance of prophylactic antibiotics as a routine [11]. Another study had revealed evidence against prophylactic antibiotics, but the number of subjects studied was small [12]. A prospective, controlled trial has tested gentamicin and chloramphenicol for pit viper envenoming in Ecuador's Amazon rain forest [13]. This study did not show a significant difference in the number of infectious complications between the treated group and the untreated group. Although the number of studies is limited, they gave similar results similar to the findings and conclusions of our study.

Our findings suggest that cytotoxin-mediated inflammation rather than bacterial infection is the leading factor in the pathogenesis of the local inflammatory swelling in snakebite. However, in view of the alarming range of bacteria recovered from the venom and oral cavities of snakes in previous studies, bacterial contamination of the bitten site may be occurring [7-9]. These bacteria may not be pathogenic to human tissues. Furthermore, the antibacterial activity of the snake venom itself has been postulated to control local infection [7]. Further studies are needed to unravel these doubts about the oral microbial flora of snakes and its ability to infect human tissues. We conclude that the routine use of antibiotics (penicillin and metronidazole) does not seem to be of value in reducing the local inflammatory swelling in venomous snakebite.

\section{References}

1. Kularatne SAM. Clinical profile of snake envenoming: A study in North Central Province of Sri Lanka. The $23^{\text {rd }}$ Bibile Memorial Oration in 2001. Sri Lanka Journal of Medicine 2001; 10: 4-12.
2. Kularatne SAM. Epidemiology and clinical picture of the Russell's viper (Daboia usselii usselii) bite in Anuradhapura, Sri Lanka: A prospective study of 336 patients. Southeast Asian Journal of Tropical Medicine and Public Health 2003; 34: 855-62.

3. Kularatne SAM, Ratnatunge N. Severe systemic effects of Merrem's hump-nosed viper bite. Ceylon Medical Journal 1999; 44: 169-70.

4. Phillips RE, Theakston RDG, Warrell DA, Galigedara Y, Abeysekara DTDJ, Dissanayake P, et al. Paralysis, rhabdomyolysis and haemolysis caused by bites of Russell's viper (Vipera russelli pulchella) in Sri Lanka: failure of Indian (Haffkine) antivenom. Quarterly Journal of Medicine, New Series 1988; 68: 691-716.

5. Warrell DA. Snake venoms in science and clinical medicine. 1. Russell's viper: biology, venom and treatment of bites. Transactions of Royal Society of Tropical Medicine and Hygiene 1989; 83: 732-40.

6. De Silva A. Oral micro-flora of wild snakes of Sri Lanka: Preliminary investigations. Sri Lanka Journal of Medicine 2002; 11: 58.

7. Theakston RDG, Phillips RE, Looareesuwan S, Echeverria $\mathrm{P}$, Makin T, et al. Bacteriological studies of the venom and mouth cavities of wild Malayan pit vipers (Calloselasma rhodostoma) in southern Thailand. Transactions of Royal Society of Tropical Medicine and Hygiene 1990; 84: 875-79.

8. Goldstein EJ, Citron DM, Gonzales H, Russell FE, Finegold SM. Bacteriology of rattlesnake venom and implication for therapy. Journal of Infectious Diseases 1979; 140: 818-21.

9. Williams FE, Freeman M, Kennedy E. The bacterial flora of the mouth of Australian venomous snakes in captivity. Medical Journal of Australia 1954; 21: 190-3.

10. Clark RF, Selden BS, Furbee B. The incidence of wound infection following crotalid envenomation. Journal of Emergency Medicine 1993; 11: 583-6.

11. Terry P, Mackway-Jones K. Best evidence topic report: the use of antibiotics in venomous snake bite. Emergency Medical Journal 1992; 19: 48-9.

12. Kerrigan KR, Mertz BL, Nelson SJ, Dye JD. Antibiotic prophylaxis for pit viper envenomation: prospective, controlled trial. World Journal of Surgery 1997; 21: 369-73.

13. Pocock SJP. Clinical Trials, A Practical Approach. New York: John Wiley and Sons, 1995.

14. Gawarammana IB, Kularatne SAM, Dissanayake WP, Kumarasiri RPV, Senanayake N, et al. Parallel infusion of hydrocortisone \pm chlorpheniramine bolus injection to prevent acute adverse reactions to antivenom for snakebites. A randomized, double-blind, placebo-controlled study. Medical Journal of Australia 2004; 180: 20-3. 